\title{
ABORDAJE DESDE LA COMPLEJIDAD A LAS BARRERAS EN LA PRESTACIÓN DE SERVICIOS DE SALUD A CONSUMIDORES DE SUSTANCIAS PSICOACTIVAS: ESTUDIO DE CASO EN EL MODELO DE SALUD COLOMBIANO \\ APPROACH FROM THE COMPLEXITY TO THE BARRIERS IN THE PROVISION OF HEALTH SERVICES TO USER OF DRUGS: A CASE STUDY IN THE COLOMBIAN HEALTH MODEL
}

Claudia Fabiola Rey-Sarmiento ${ }^{1-4}$, Diana Rocío Bernal-Camargo ${ }^{5}$, Johan Avendaño Arias ${ }^{6}$, Gerardo González ${ }^{2-3}$ y Mauricio RomeroGonzález ${ }^{2-3}$

\begin{abstract}
${ }^{1}$ Multiversidad Mundo Real Edgar Morin, México; ${ }^{2}$ MAYU of New England, New Haven, CT, United States; ${ }^{3}$ Division of Addiction Psychiatry, University of Massachusetts, Worcester, MA, United States; ${ }^{4}$ Facultad de Administración, Finanzas y Ciencias Económicas, Universidad EAN, Colombia; ${ }^{5}$ Grupo Derechos Humanos, Facultad de Jurisprudencia, Universidad del Rosario, Colombia; ${ }^{6}$ Universidad Central, Facultad de Ciencias Sociales, Especialización en Geografía,
\end{abstract} Colombia

\section{Abstract}

Introduction. The phenomenon of the consumption of psychoactive substances (SPA) needs to be approached from the complex given that there are no linear deterministic models that can explain it. SPA use requires for its understanding, the approach from different levels of reality to better understand the barriers of access to health services. Objective. This article identifies and analyzes, from the complexity, the existing barriers of access to services for SPA users by health providers in the Colombian Health System. Methodology. This is a transdisciplinary and qualitative research, involving providers of the health system treating people with SPA. Three regions were selected. In each region, technical assistance, social cartography, focus group and thematic questionnaire were performed. Results: Barriers of access to services were identified that included administrative barriers in differential approach, and recognition of human rights of SPA consumers as subjects of special protection. Conclusions. Its recommended to understand the problem of SPA from an interdisciplinary perspective that can allow to enhance at the local level, the human rights approach in the provision of health services to SPA consumers.

Keywords: Complexity, use of drugs, access barriers, Colombian health model, human rights approach

\section{Resumen}

Introducción. El fenómeno del consumo de sustancias psicoactivas (SPA) se aborda desde lo complejo, por cuanto no puede ser explicado por modelos lineales de determinación, y se postula que requiere para su comprensión la aproximación a diferentes niveles de realidad, desde esta perspectiva se propone estudiar las barreras de acceso a los servicios de salud. Objetivo. Evidenciar y analizar, desde la complejidad, las barreras existentes en el sistema de salud colombiano, por parte de los prestadores de servicio de salud, en la atención de las personas con trastornos por el uso de sustancias y su comorbilidad asociada. Metodología. Se trata de una investigación transdisciplinar y cualitativa, en la que participaron agentes del sistema de salud relacionados con el consumo de SPA, agrupados en tres regiones de Colombia. Se efectuó una asistencia técnica por región, aplicando cartografía social, grupo focal y cuestionario temático. Resultados: Se encontraron barreras en relación con aplicación de enfoque diferencial, reconocimiento y garantía de consumidores de SPA como sujetos de especial protección, y otras administrativas. Conclusiones. Acorde con los hallazgos, se requiere comprender el fenómeno del consumo de SPA desde una mirada transdisciplinar, que permita aunar esfuerzos en lo local, para efectivizar el enfoque de derechos en la prestación de servicios de salud hacia consumidores de SPA.

Palabras clave: Complejidad, Barreras de acceso, consumo de sustancias psicoactivas, modelo de salud colombiano, enfoque de derechos humanos

Correspondencia: fareysar@gmail.com 
Los fenómenos de salud han sido históricamente explicados en la medicina, por modelos lineales, de causa - efecto, determinísticos, tales como el modelo de Historia natural de la enfermedad o las determinantes de la salud; sin embargo, es necesario reflexionar estos fenómenos desde una óptica diferente, que no implique una relación directa, entre la causa y el efecto y, como propone Ramis (2007), permitirse una nueva forma de pensarlos sin reducir el todo a las causas, desde la complejidad, entendida esta, como propone Maldonado (1999. en Sotolongo y Delgado, 2006), en tres líneas principales de trabajo: como ciencia que estudia la dinámica no lineal (los efectos no siguen directamente las causas); como forma de pensamiento que supera las aproximaciones disciplinares y dicotómicas (salud enfermedad); y como una nueva mirada que supera el reduccionismo.

Por ello, este documento propone una aproximación al fenómeno del consumo de sustancias psicoactivas como un fenómeno complejo, entendiendo que los efectos que se producen en salud no responden de forma directa y proporcional a la causa (ruptura de la simetría) y por ello, se requiere para su comprensión la aproximación a diferentes niveles de realidad; esta síntesis de la investigación, ha sido realizada obedeciendo a esta aproximación conceptual propuesta por Naomar (2006), contemplando en ella, las perspectivas de los agentes del sistema general de seguridad social en salud - SGSSS en Colombia.

De otra parte, la Resolución UNGASS 2016, plantea la necesidad de un enfoque de derechos donde se incorporen compromisos frente a la no estigmatización, no discriminación y la generación de estrategias y políticas para promover la garantía del acceso a los tratamientos, teniendo como principio fundamental el consentimiento informado de los consumidores de sustancias psicoactivas (SPA); abordando el fenómeno desde la política criminal. Otro abordaje es desde la problematización del mismo, tal como lo plantean (Camarotti y Kornblit 2015), "a pesar de los esfuerzos gubernamentales, de organizaciones de la sociedad civil y de los particulares destinados a encarar el consumo problemático de drogas en el mundo y de los recursos invertidos en las respuestas implementadas, los resultados obtenidos hasta la fecha han sido magros. (...) producto de las políticas que en materia de lucha contra las drogas se han venido llevando a cabo"; siendo estas algunas de las miradas lineales a este fenómeno.

El Estado Colombiano ha diseñado e implementado instrumentos normativos, para el cumplimiento de estándares internacionales y jurisprudenciales relacionados con el desarrollo y materialización efectiva del derecho a la salud para la atención de las personas con trastornos relacionados con sustancias y trastornos adictivos. El Ministerio de Salud y Protección Social - MSPS en Colombia, a través de las diferentes normas expedidas busca dar respuesta a las necesidades de atención identificadas con relación al consumo de sustancias psicoactivas, reconociendo el consumo, abuso y adicción a sustancias psicoactivas, lícitas o ilícitas como "un asunto de salud pública y bienestar de la familia, la comunidad y los individuos(...)" en la Ley 1566 de 2012, esta misma norma define que estos deberán ser tratados como una "enfermedad que requiere atención integral por parte del Estado, conforme a la normatividad vigente y las Políticas Públicas Nacionales en Salud Mental y para la Reducción del Consumo de Sustancias Psicoactivas y su Impacto, (...)" (Congreso de la República, 2012)

Esta norma, tiene como gran desafío para el fortalecimiento de la política de drogas el abordaje del consumo de sustancias psicoactivas- SPA en Ios diferentes niveles de intervención, así como en las distintas dimensiones de la política pública, pues al considerar el consumo desde el ámbito de la salud pública, le permite tener mayor injerencia frente a la materialización de los derechos y la protección de los usadores de sustancias en el marco del SGSSS.

De forma complementaria, el máximo tribunal constitucional colombiano, en relación con los sujetos con farmacodependencia ha establecido que ellos "tienen un sistema de protección especial que se ve reforzado por su condición de manifiesta debilidad psíquica, que obliga al Estado y a sus entidades a garantizar una protección y un tratamiento integral para superar dicha patología". (Corte Constitucional, 2012)

Por tanto, según esta misma institución es obligación de las aseguradoras

“(...) brindar a sus afiliados que padecen trastornos mentales derivados del consumo, abuso o adicción a sustancias psicoactivas lícitas o ilícitas, los servicios de salud incluidos en el entonces denominado Plan 
Obligatorio de Salud - POS- dirigidos a tratarlos. No hacerlo cuando lo requieren, en el sentido de que el médico tratante adscrito a la entidad lo ordena, les vulneraría el derecho fundamental a la salud. Igualmente, cuando se trata de procedimientos ordenados por el médico tratante para superar adicciones no incluidos en el POS, las Entidades Prestadoras de Servicios de Salud EPS- tienen la obligación de cubrirlos si son necesarios, dado que la persona no cuenta con los recursos económicos para costearlos" (Corte Constitucional, 2012)."

Con relación a las barreras de tipo administrativo, en sentencia T-153 de 2014, la Corte ha sido enfática en señalar que este tipo de pacientes con dependencia a SPA, no deben someterse a cargas administrativas, para la consecución efectiva del tratamiento requerido, conforme a sus preferencias y necesidades, dada su situación de debilidad manifiesta (Corte Constitucional, 2014).

Por lo anterior, en esta investigación se pretende abordar la atención al fenómeno de la cultura de las drogas, término acuñado por Musto (1993), desde las ciencias de la complejidad y desde un acercamiento transdisciplinar, que incluya, además de los niveles de percepción de los agentes del sistema, la atención clínica o las barreras desde la óptica de los usuarios, y la percepción desde la institucionalidad; como propone Peláez desde un "modelo sociocultural, donde, desde la investigación cualitativa en ciencias sociales, se focaliza en la importancia de la cultura y el contexto social" (2016). Para lograr lo anterior, se utilizaron herramientas de diversas disciplinas, que permiten una mirada integradora de la prestación de servicios de salud al usador de SPA, como una variable fundamental del sistema abierto, del consumo de SPA.

\section{MÉTODO}

Con el propósito de brindar un marco suficiente a la construcción de política pública, basada en evidencia, se realizó un proceso investigativo del fenómeno, abordándolo desde una mirada transdisciplinar que conjuga herramientas de las ciencias sociales, como la cartografía social; de las ciencias administrativas y de las ciencias de la salud, comprendiendo el fenómeno desde los servicios de salud necesarios para la garantía de la atención de las personas, estableciendo un diálogo intersaberes en la síntesis final de los conocimientos propuestos, para dar cuenta de forma operativa de nuevas formas de saberes con relación a los fenómenos de salud y, en particular del fenómeno de consumo de SPA.

La cartografía social entendida como esa estrategia de diálogo entre los actores, busca que quienes viven y construyen el territorio y sus relaciones de territorialidades, sean quienes narren a través de la graficación, cada una de evidencias de tensiones, acuerdos, problemáticas y alternativas en una figura hecha a muchas manos. Se trató entonces de ofrecer unas preguntas orientadoras a ser respondidas gráficamente en las figuras para luego a manera de cierre, tener una socialización sobre los resultados obtenidos.

Para determinar las barreras en la prestación de servicios, se propuso una metodología cualitativa que involucrara a determinados agentes del sistema de salud relacionados con la prestación de servicios en materia de consumo de SPA: Entidades Territoriales ET, Secretarías de Salud, Aseguradoras de los diferentes regímenes, prestadores de salud IPS, Profesionales de la Salud, sector académico, organizaciones sociales, fundaciones y demás agentes que participan de manera directa o indirecta en la atención al consumidor de sustancias Psicoactivas.

El contacto de hizo a través del Ministerio de Salud y la Protección Social - MSPS y la Organización de Naciones Unidas contra las Drogas y el Delito - UNODC, para que, priorizando departamentos de tres regiones del país (Eje Cafetero, Zona Norte y Centro), se realizaran asistencias técnicas en las cuales se desarrollaron mesas de trabajo, se aplicó un cuestionario de recolección de información y una cartografía temática y social, a fin de lograr identificar la población y /o usuarios afectados por el consumo de las sustancias psicoactivas, los servicios ofertados por departamento, teniendo en cuenta la demanda y oferta, las barreras de acceso a los servicios de Sustancias Psico Activas -SPA y la garantía de derechos.

En lo relacionado con la prestación de servicios de salud para consumo de SPA, se utilizó como fuente primaria, los Registros Especiales de Prestación de Servicios - REPS, consultada en la página del MSPS, así 
como la información suministrada por las entidades aseguradoras y prestadoras del SGSS en asistencias técnicas.

Las discusiones se guiaron por un conjunto de preguntas diseñadas y validadas previamente a fin de obtener evidencias e información técnica junto con las experiencias y percepciones de los participantes respecto a la temática abordada. Las preguntas orientadoras contenidas en el instrumento de recolección de información sobre prestación de servicios y derechos, permitió recolectar información cualitativa, ya que la muestra no fue suficiente para hacer un análisis cuantitativo.

Como se irá mostrando en los resultados, las cartografías construidas conservaron el enfoque diferencial y poblacional para tener un primer perfil de los consumidores de SPA en las 3 regiones. Este permitió visibilizar los patrones territoriales, las tendencias, las poblaciones más afectadas en términos de consumo, el riesgo paulatino que se ha venido presentando en grupo que según los estudios anteriores no eran potenciales consumidores; resaltando el hecho que al parecer la homogenización del SPA es cada vez mayor territorialmente, donde los municipios y/o regiones pequeñas ya no están exentos pues poblaciones desplazadas, mujeres en ejercicio de prostitución, habitantes de calle, miembros de la fuerza pública, etc, solo por mencionar algunos ejemplos, son identificados como consumidores.

Las cartografías ponen en evidencia el rol de los nodos urbanos, a partir de los cuales pareciese que se ampliara la dispersión del SPA hasta el punto que se generan áreas de influencia territoriales por perfiles particulares. Así, siendo este un primer ejercicio, se aporta en la construcción de una línea base para la geografía del consumo de SPA en Colombia.

Para las categorías I y II de análisis y presentación de resultados, se aplicaron técnicas de grupo focal, cuestionario auto-diligenciado y cartografía social.

\section{RESULTADOS}

Los resultados se presentaron y analizaron en las siguientes categorías:
I. Identificación de población afectada por consumo de SPA

II. Reconocimiento y la garantía a los derechos de los usuarios consumidores de SPA.

III. Barreras en la prestación efectiva de servicios de salud hacia este grupo poblacional.

La información se presenta agrupada en las tres zonas, tomando los departamentos que participaron, así: Eje Cafetero: Caldas, Quindío y Risaralda; Eje Centro: Meta, Tolima, Cundinamarca, Santander, Norte de Santander y Bogotá; y Eje Costa Caribe: Atlántico, Bolívar, Guajira, Sucre, Magdalena y Cesar.

\section{Identificación de población afectada por consumo de SPA}

En la zona Eje Cafetero, como se observa en las figuras de cartografía, los participantes identificaron una prevalencia de población consumidora de SPA en condiciones de desplazamiento y en menor proporción de población con $\mathrm{VIH}$, con discapacidad y condiciones combinadas (Figura 1). Denotan mayor consumo en población de Niños, Niñas y Adolescentes (NNA) y comunidad LGBTI (Figura 2), y sin mayores distinciones proporcionales entre población del régimen contributivo o del subsidiario (Figura 3).

Así mismo, identificaron una prevalencia, en términos de ocupación, de población estudiante frente a docentes, miembros de la fuerza pública, desempleados o condiciones combinadas (Figura 4) y en relación con el origen étnico, a la población indígena y afrodescedendiente (Figura 5), concentrados en zonas alejadas, que ingresaron al consumo por razones de orden social como el desempleo, la falta de oportunidades y el tráfico de drogas.

En esta misma categoría población, en la figura se identificó prevalencia de consumo de SPA, en la zona centro del eje cafetero, entre población habitante de calle, en ejercicio de prostitución y privada de la libertad (Figura 6). (Grupo Focal, asistencia técnica No.1) 


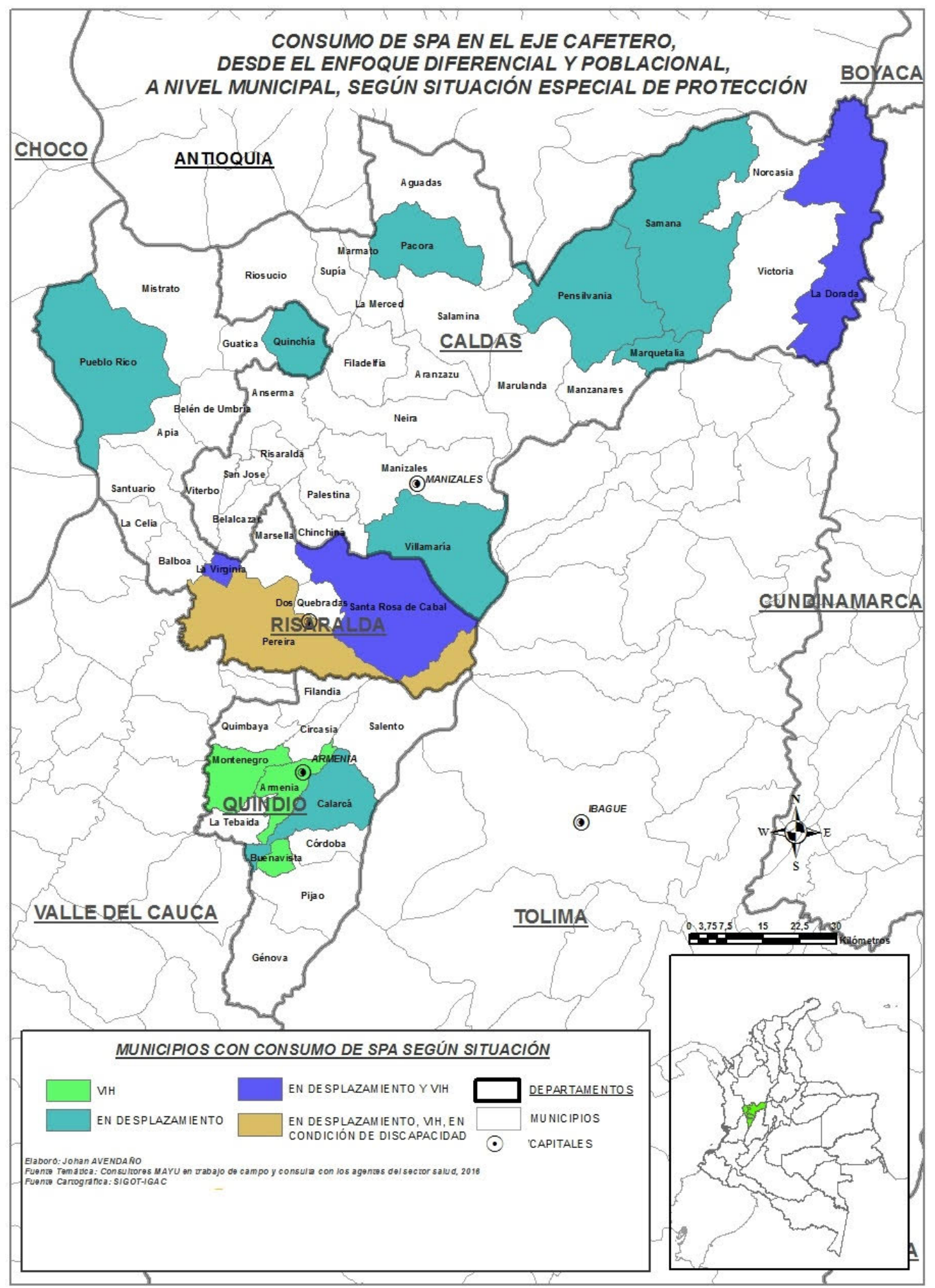

Figura 1. Identificación población consumidora de SPA desde enfoque diferencial y poblacional según situación especial de protección en Eje Cafetero. Fuente propia 


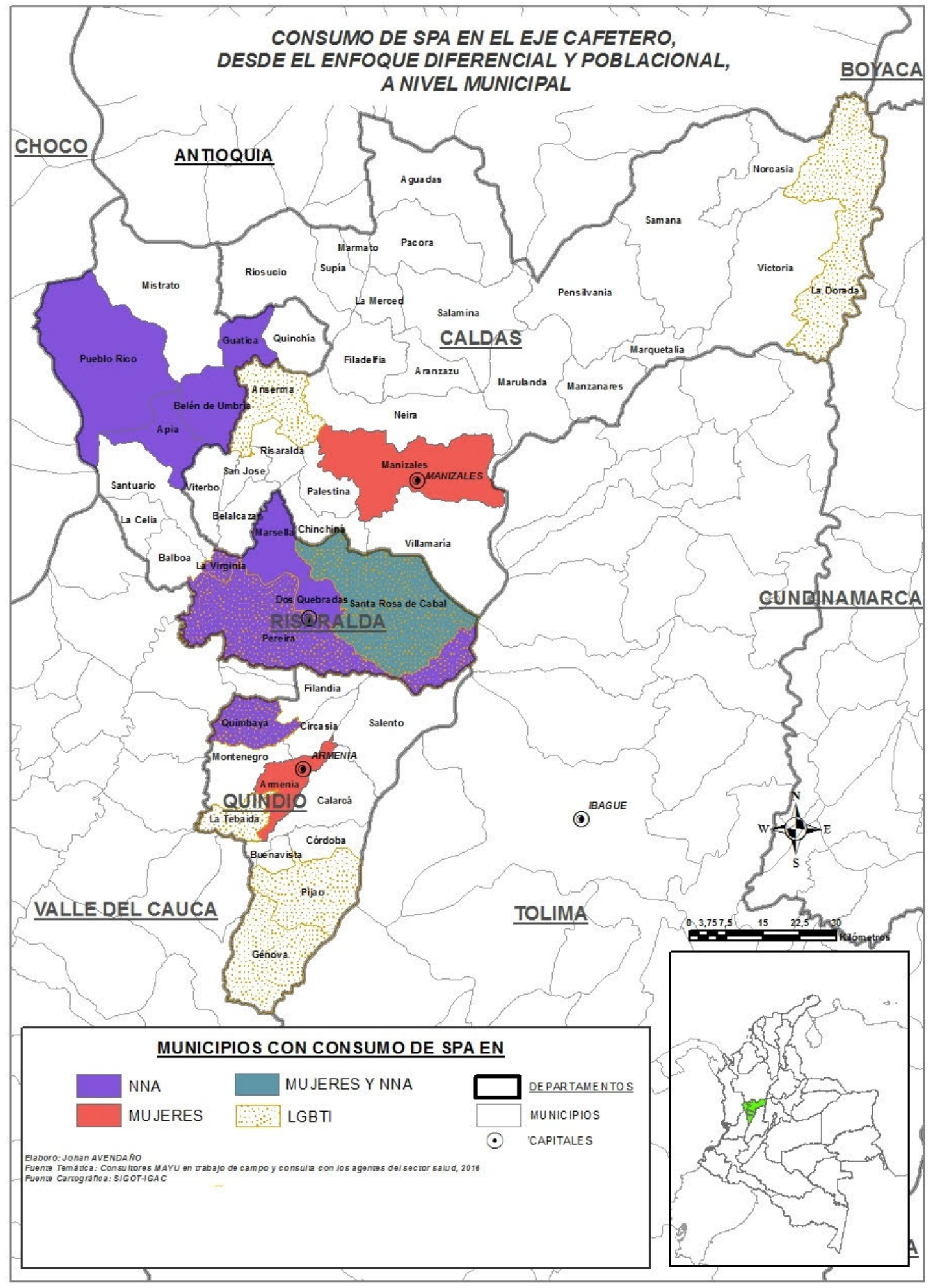

Figura 2. Identificación población consumidora de SPA desde enfoque diferencial en Eje Cafetero. Fuente propia 


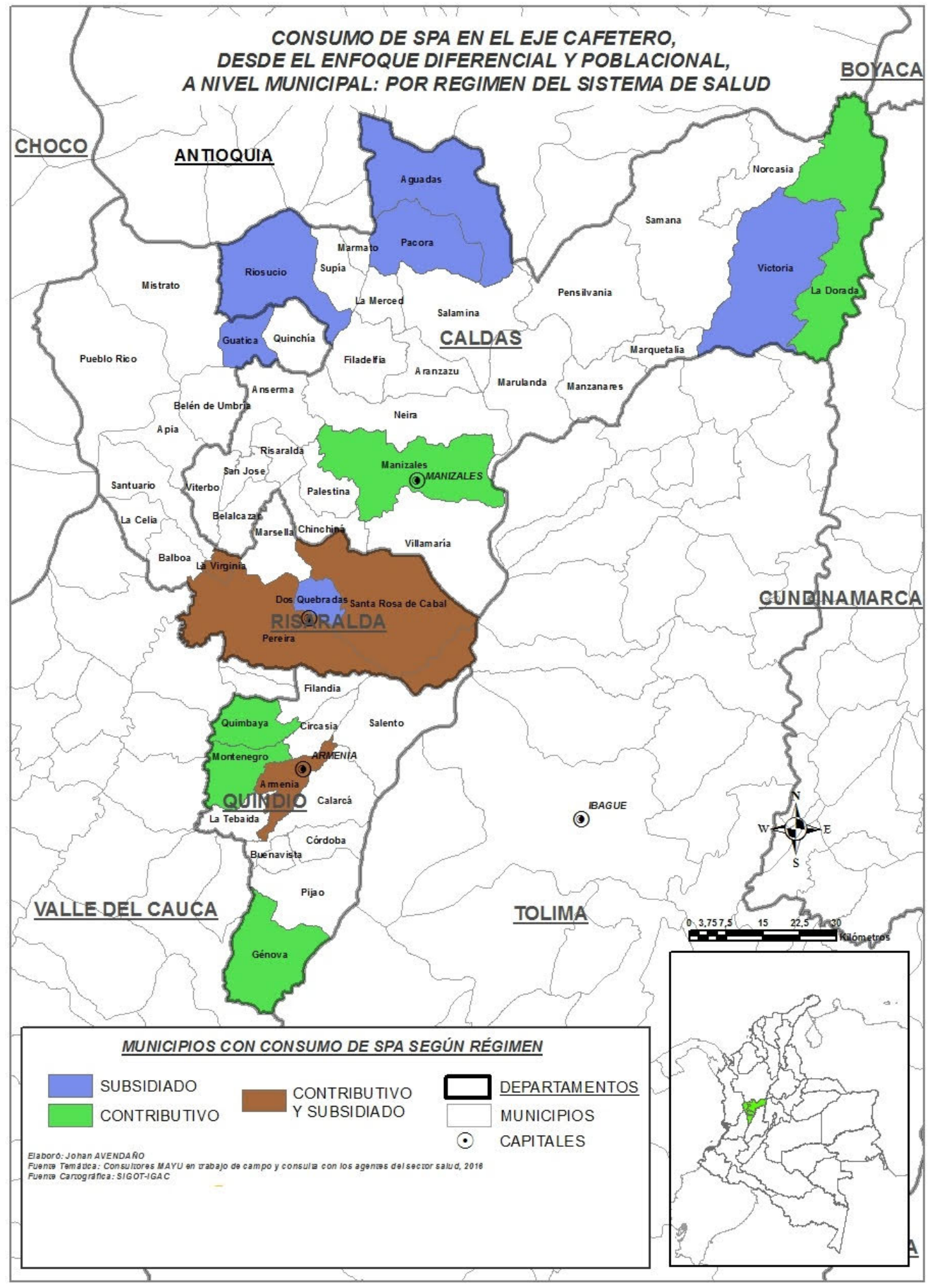

Figura 3. Identificación población consumidora de SPA según el régimen de salud en Eje Cafetero. Fuente propia 


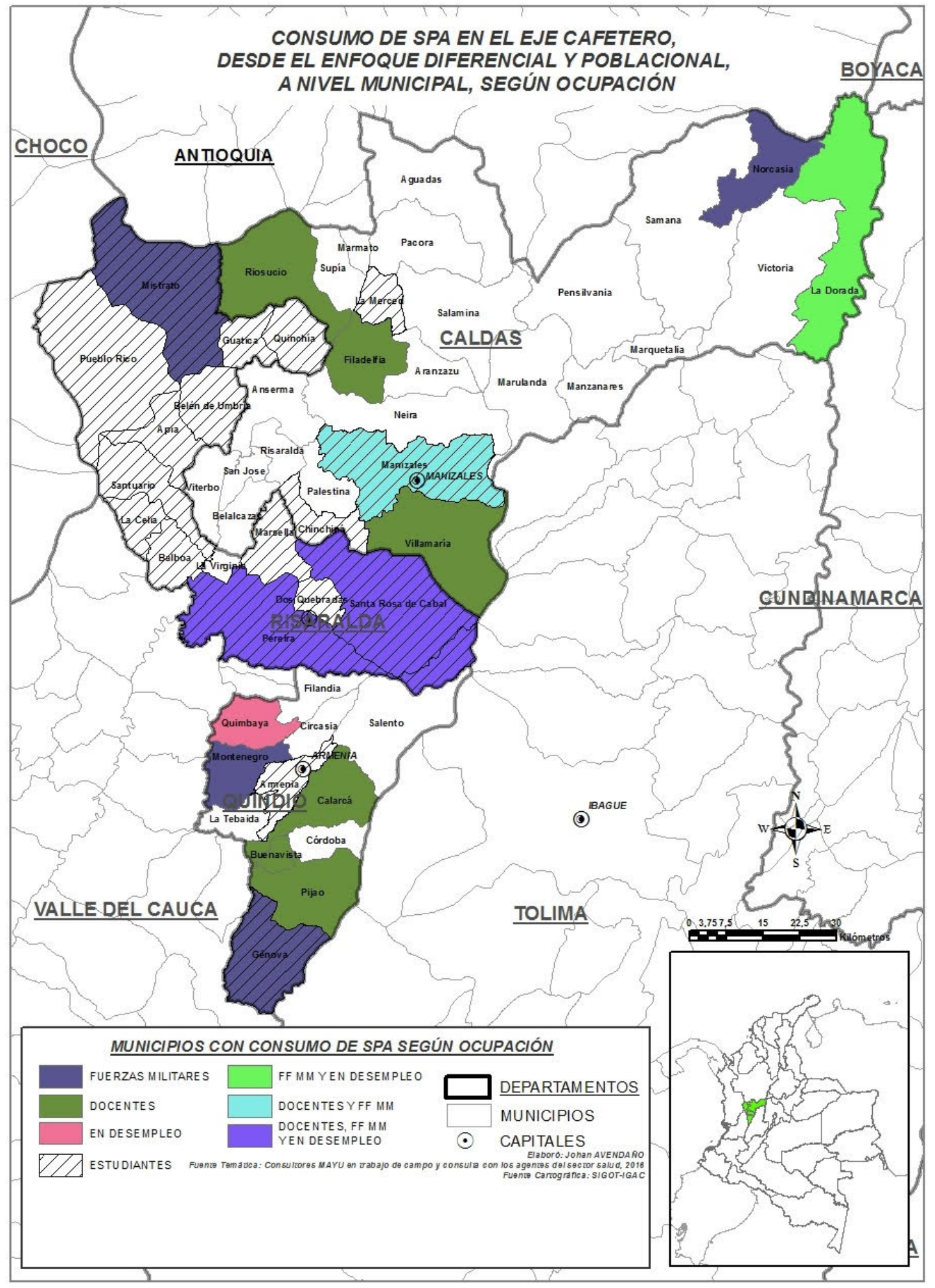

Figura 4. Identificación población consumidora de SPA por ocupación prevalente en Eje Cafetero. Fuente propia 


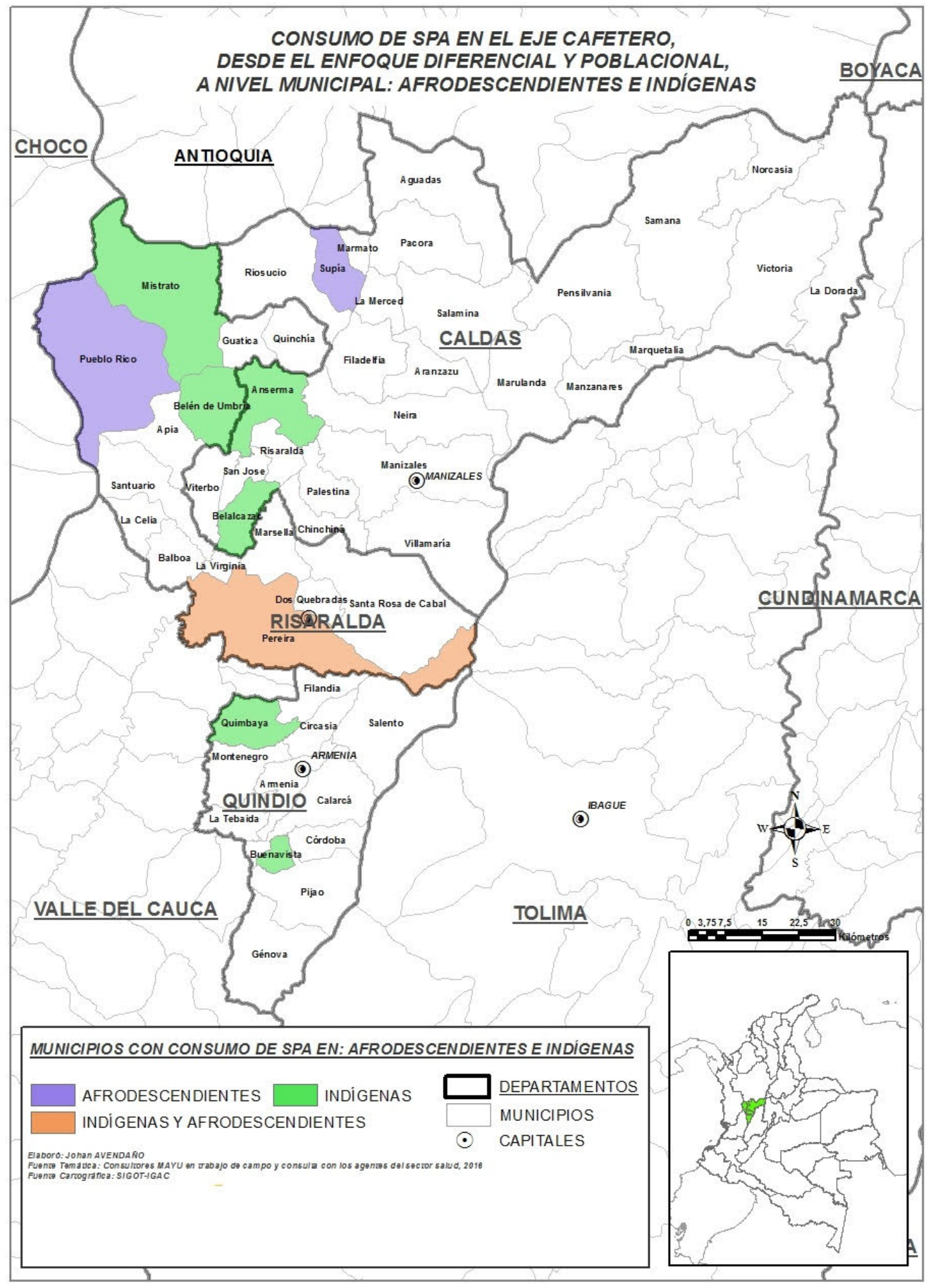

Figura 5. Identificación población consumidora de SPA por origen étnico. Fuente propia 


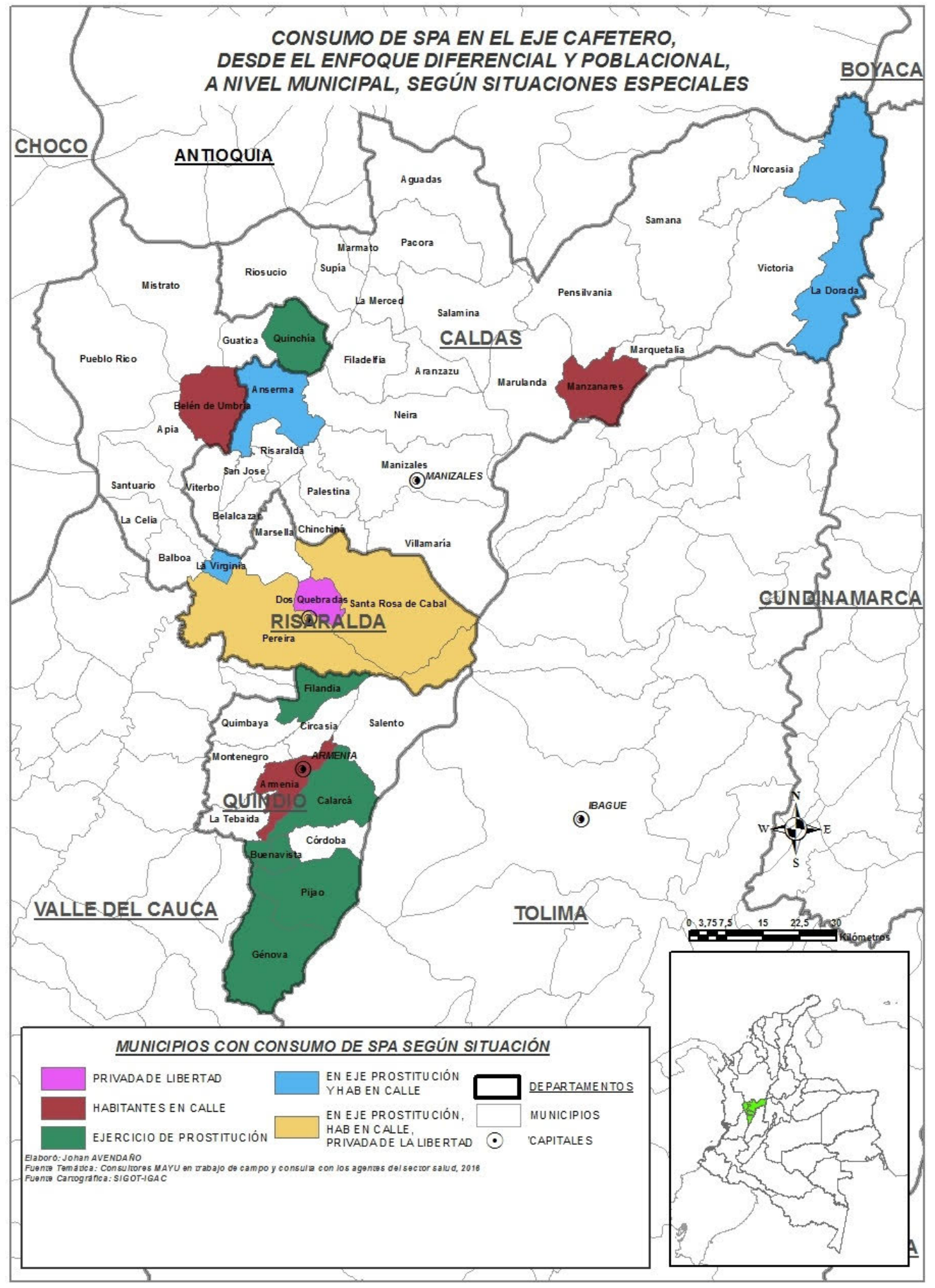

Figura 6. Identificación población consumidora de SPA con determinantes de salud en Eje Cafetero. Fuente propia 
En cuanto a la zona centro, en términos generales los participantes señalaron que los usuarios y familias de consumidores de SPA, pertenecen tanto al régimen contributivo como al subsidiado, aunque también tienen usuarios particulares en algunas zonas, especialmente se evidenció en el caso de Norte de Santander, que a su vez resaltó la presencia de usuarios del régimen subsidiado en el municipio de Tibú y cercanos, lo mismo se manifestó en el caso del departamento de Santander en donde hay prevalencia usuarios de este régimen en los municipios de Málaga y San Gil.

Si bien en el desarrollo del grupo focal se evidenció la preocupación por el consumo por parte de la población en condiciones de desplazamiento en la generalidad del territorio, para el caso de Norte de Santander se encontró una percepción de mayor preocupación para el caso de Cúcuta, agregando el caso de la población flotante consumidora, que particularmente viene de Venezuela. Por su parte, para el caso de Santander, se puso de manifiesto esta condición para el caso de municipios como Barrancabermeja y Bucaramanga.

Por su parte, los asistentes de Bogotá, señalaron que precisamente la capital por ser receptora por excelencia de población desplazada, tiene como problemática dentro de la misma, el consumo de SPA, situación que se ha evidenciado en diferentes informes sobre desplazamiento en la ciudad.

Se evidenció una especial una preocupación en relación con el consumo en NNA escolarizados y no escolarizados, por lo que los asistentes ubicaron esta población en cada uno de los departamentos objeto de la cartografía.

Igualmente, manifestaron que en efecto existe una caracterización en términos la diversidad de género, con percepción de alto riesgo en la población LGBTI, pero no se evidencia en lo práctico como se efectiviza esta diferenciación en la ruta de atención específica.

En relación con la profesión u oficio señalaron una preocupación especial por determinados grupos a los que no se accede con facilidad ni a usuarios ni a información detallada, como lo es el caso de miembros de la Fuerza Pública, de manera especial se señaló en este grupo para el caso de Norte de Santander, Tolima y Meta, en donde a su vez, la presencia del ejército "consideran que ha traído consigo el consumo de sustancias", pese a que no existen datos de evidencia. (Grupo Focal, asistencia técnica No.2)

Acorde con la información suministrada en el marco de las discusiones del grupo focal y la cartografía, los participantes de Bogotá manifestaron la problemática de consumo de SPA relacionada a poblaciones y grupos étnicos con presencia en la ciudad, y en particular entre aquellos que adicionalmente han sido víctimas con ocasión de desplazamiento forzado en el país.

En departamentos como Santander y Norte de Santander, se evidencia la relación entre consumo de SPA y grupos étnicos no en todo el departamento sino en algunas de sus regiones. Como se mencionó anteriormente, para el caso de las personas dedicadas al ejercicio de la prostitución, existe una relación con presencia de miembros de la fuerza pública e incremento de turismo.

Por su parte, en el caso de Bogotá, se evidenció la problemática relacionada con el consumo entre habitantes de la calle y el impacto de la intervención distrital en el llamado sector del Bronx, generando un desplazamiento de estas personas, tanto al interior de la ciudad como hacia municipios aledaños dentro y fuera del departamento de Cundinamarca (Figura 7).

En la zona norte costa caribe, como se observa en los mapas de cartografía, los participantes identificaron una prevalencia de población consumidora de SPA en condiciones de desplazamiento y de población con $\mathrm{VIH}$ (Figura 8).

Conforme al análisis de los agentes, se encontró que existen grupos consumidores de indígenas en zonas de difícil acceso, y denotan mayor consumo en población de Niños Niñas y Adolescentes (NNA) estudiantes (Figura 9).

\section{Reconocimiento y la garantía a los derechos de los usuarios consumidores de SPA}

En desarrollo de las asistencias técnicas para la valoración y orientación sobre los derechos y mecanismos de exigibilidad para la atención del consumo de SPA, se consultó a los agentes del sistema, organizaciones y demás instituciones vinculadas a la problemática del consumo sobre los niveles de reconocimiento y efectividad de los derechos. 


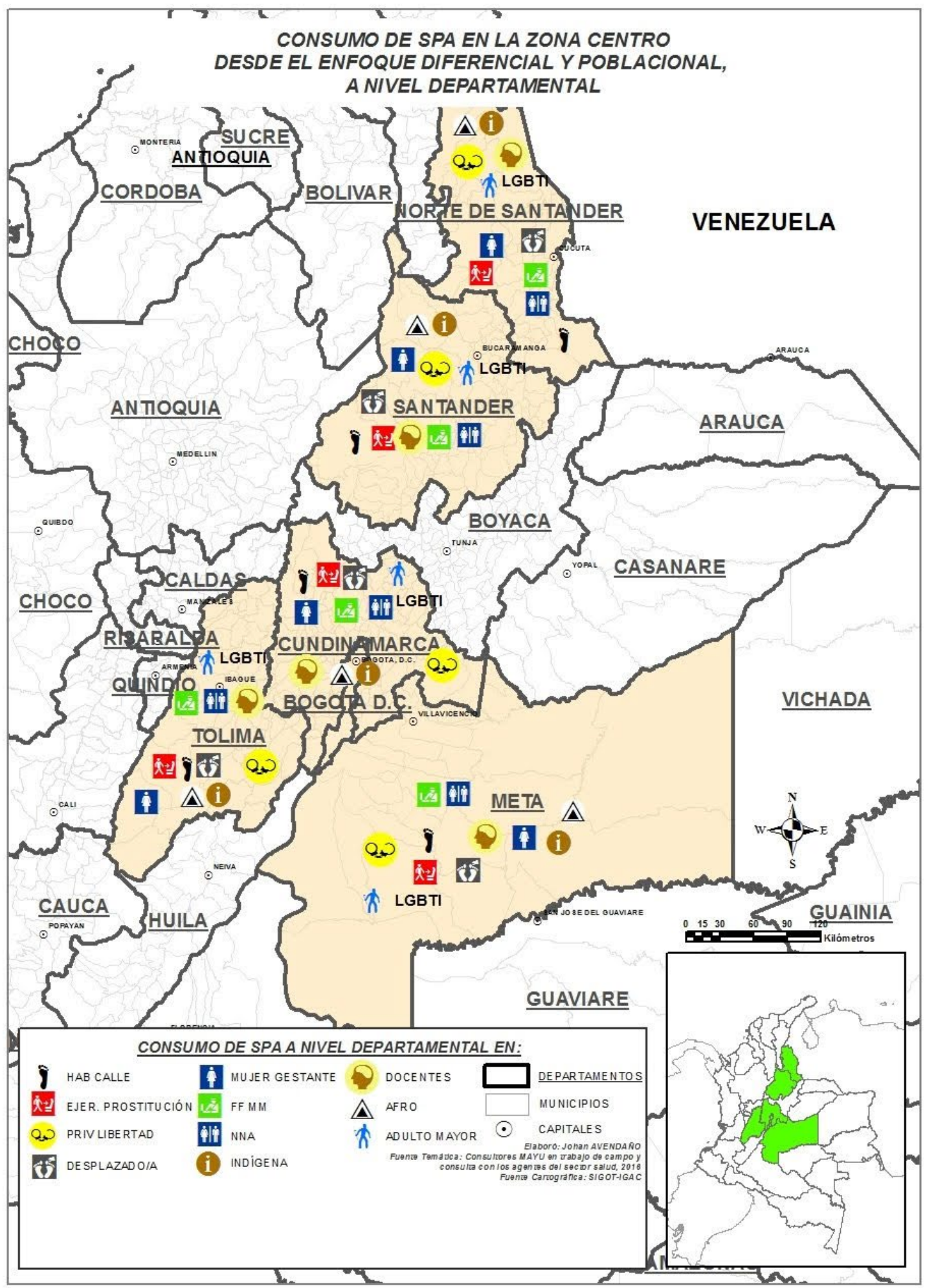

Figura 7. Identificación de poblaciones conforme a los enfoques establecidos en el PDSP en la Zona Centro. Fuente propia 


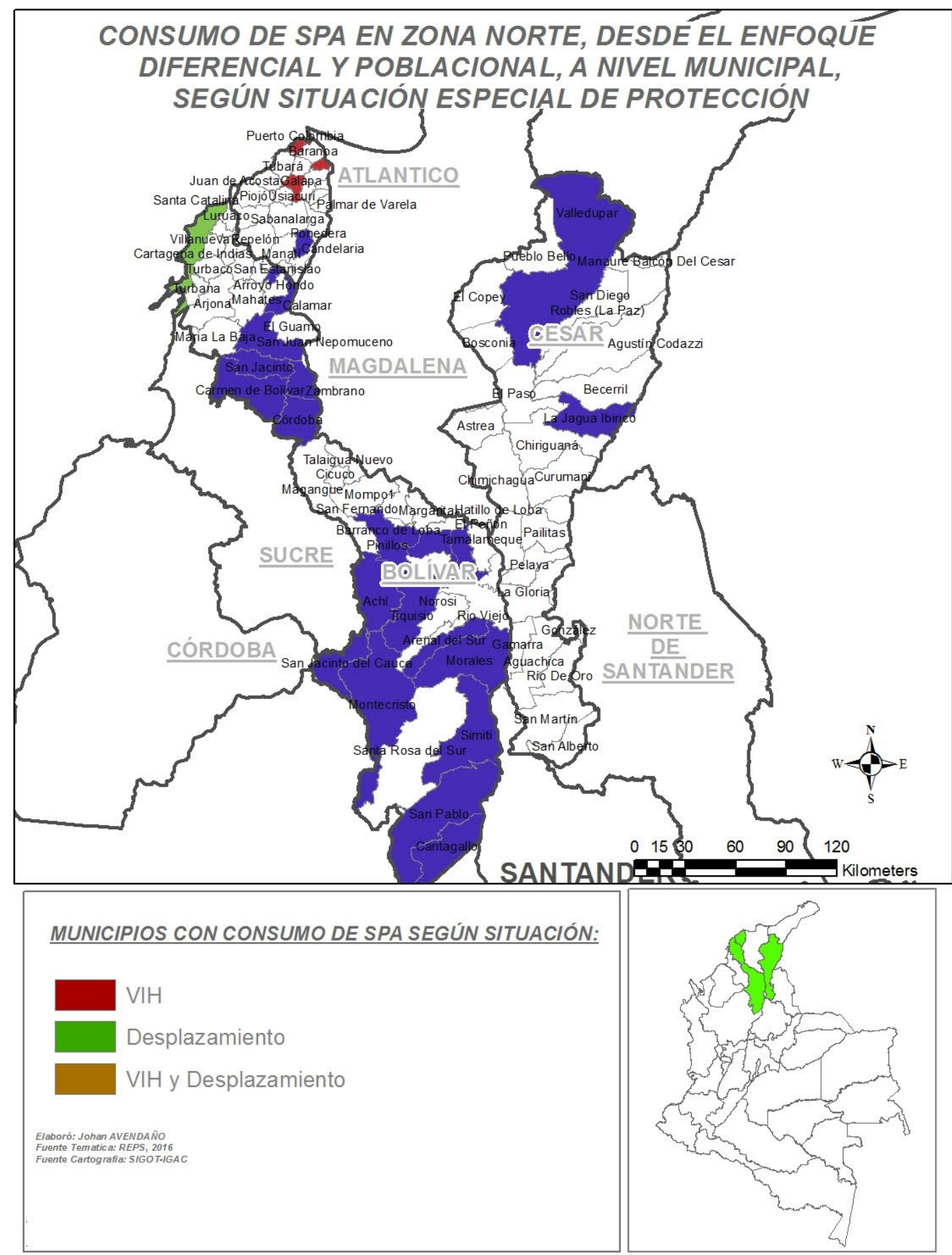

Figura 8. Identificación de poblaciones diferenciadas en situación de consumo de la Zona Norte. Fuente propia 


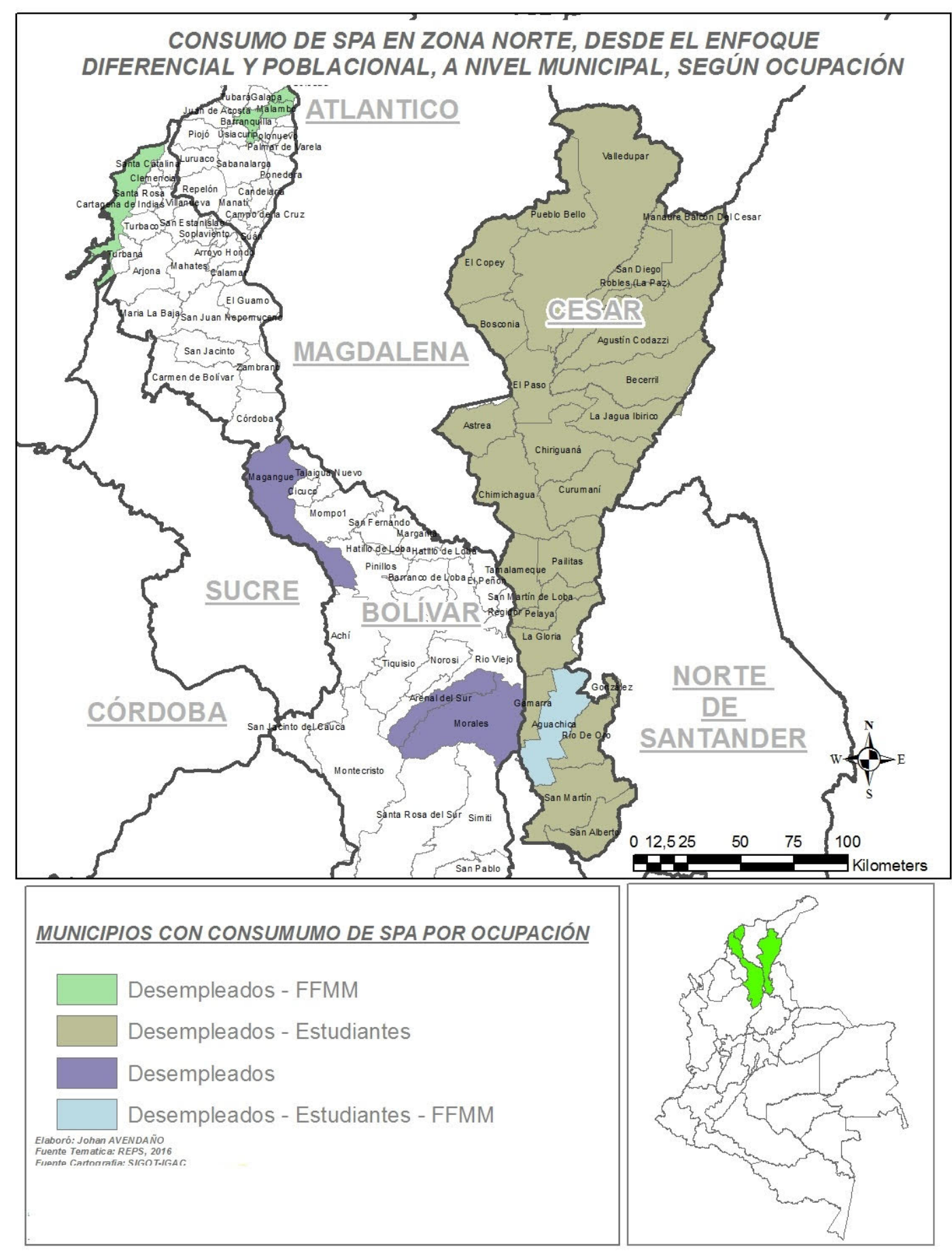

Figura 9. Identificación de poblaciones diferenciadas en situación de consumo de la Zona Norte. Fuente propia 
Como punto de partida de análisis se consideraron los derechos a la información y conocimiento, participación e inclusión social, a la intimidad y protección de datos, a la prestación de servicios de salud y sobre cuáles han sido los mecanismos de exigibilidad más efectivos para la prestación de los servicios de salud. Las zonas Cafetera, Oriente, Centro y Norte consultados en su mayoría calificaron como bajo el nivel de aplicación de los derechos anotados (Figura 10).

Se consideró que en todo se hacía reconocimiento de estos a través de los decálogos o las cartas de derechos de los pacientes que lo incluían, como el derecho a la información (en términos de información general de servicios e individual a través del consentimiento informado), el derecho a la intimidad, a la privacidad y a la prestación en sí misma de servicios de salud (Tabla 1).

\section{Barreras en la prestación efectiva de servicios de salud hacia este grupo poblacional}

A partir de los tres grupos focales realizados, uno en cada una de las zonas previamente priorizadas, se identificaron las siguientes barreras específicas para la prestación de servicios de salud requeridos por los usadores de SPA desde una perspectiva enfocada en la garantía del derecho a la salud de este grupo en situación de vulnerabilidad.

\section{Desconocimiento de la normatividad vigente}

La normatividad vigente se presenta como una fortaleza que respalda la prestación de los servicios de salud requeridos por los usuarios de SPA, pero existe un desconocimiento de ésta y de las acciones que están bajo responsabilidad del aseguramiento, lo que a genera una barrera para esta población. A esto, se aúna la desarticulación entre los diferentes agentes responsables de estos servicios, la no inclusión de tecnologías que favorecen la atención a los usuarios con consumo y el reconocimiento por parte de los agentes responsables del desconocimiento de la normativa (v.gr. funcionamiento operativo de la norma, plan de intervenciones colectivas, plan decenal de salud pública y

\footnotetext{
1 El Modelo Integral de Atención en Salud - MIAS es el mecanismo mediante el cual se implementa la Política de Atención Integral en Salud en Colombia - PAIS, el modelo cuenta con herramientas operativas
}

su implementación en el contexto territorial). Esta situación genera:

1. La no realización de acciones conjuntas buscando el cumplimiento de las mismas frente al derecho a la salud y su garantía en esta población, conforme lo dispuesto en la Ley Estatutaria;

2. Incremento de la vulnerabilidad en salud de la población afectada;

3. Aumento del riesgo en los procesos específicos contratación, por asimetría de información y poder entre aseguradores y prestadores, y

4. No atención oportuna de la demanda existente.

Limitada interacción entre los planes de beneficios (PIC POS)

Los grupos focales permitieron evidenciar un desconocimiento del Plan de Intervenciones Colectivas PIC y las actividades contenidas en el Plan Decenal de Salud Pública (MSPS, 2013) y la Resolución 0518 de 2015 (MSPS, 2015), lo que genera una amenaza en la articulación e integralidad de los servicios de salud, en el marco del actual Modelo Integral de Atención en Salud MIAS ${ }^{1}$.

Acorde con lo expresado, existe poco o nulo seguimiento a los servicios que se prestan en la entidad territorial, limitada interacción entre el PIC y el POS, pobre oferta de servicios especializados en relación con la demanda de los mismos, problemas en la contratación y facturación de los servicios, falta de información a los usuarios sobre la red de servicios y la ausencia de las entidades responsables de la vigilancia y control.

De otra parte, la integralidad de la atención y la operatividad de la Ruta Integral de Atención en Salud RIAS- para consumo de SPA se ve afectada en los territorios, por no contar con instituciones fortalecidas que estén trabajando en promoción, prevención y reducción del riesgo como respuesta al plan de drogas, lo que genera una barrera en la integralidad de la atención a la población con consumo de SPA.

mediante las cuales se instrumentaliza, una de estas herramientas son las Rutas Integrales de Atención definidas y priorizadas, una de las cuales da cuenta de la atención al consumo de SPA. 


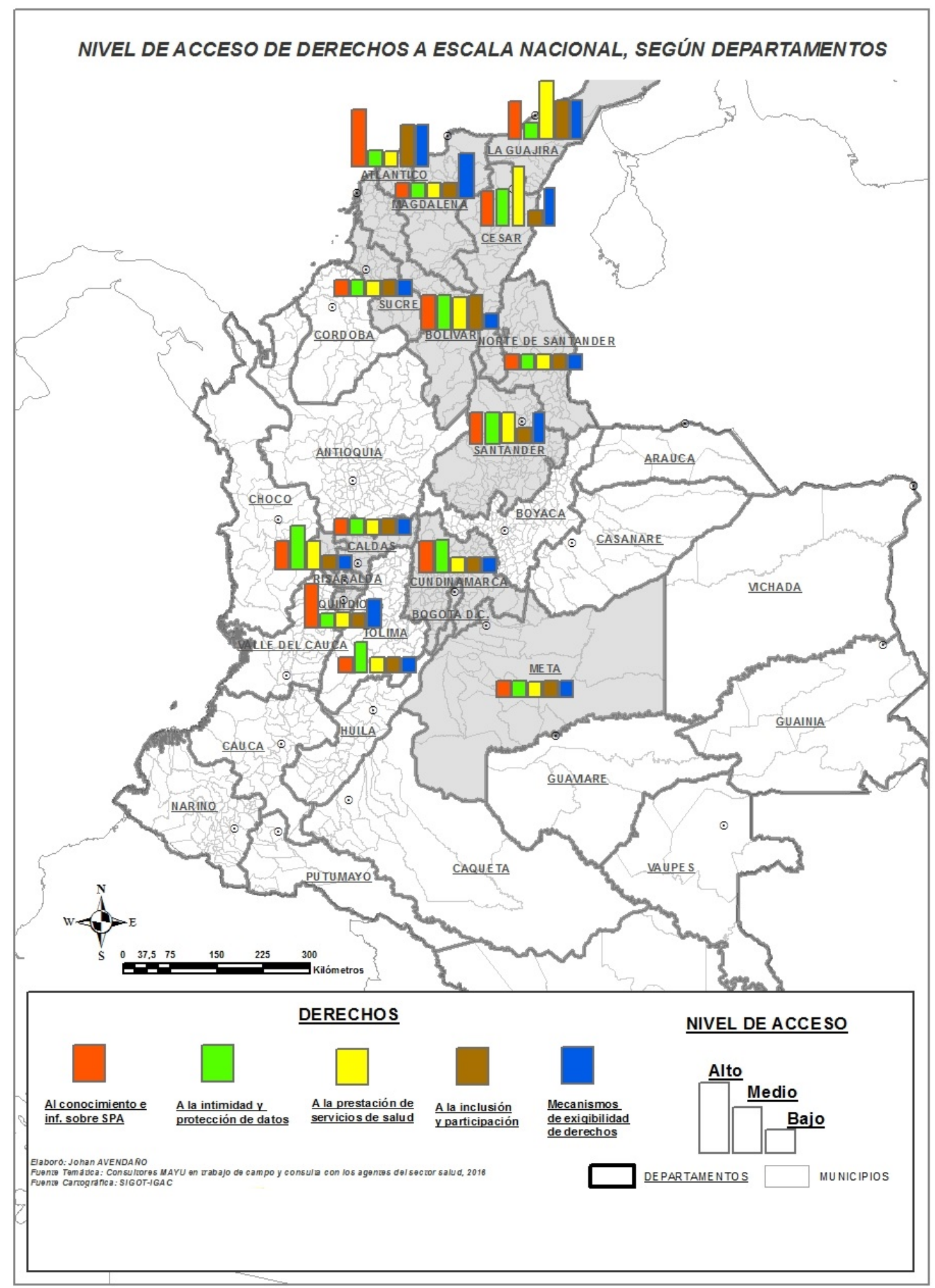

Figura 10. Reconocimiento y acceso a derechos por parte de consumidores de SPA. Fuente propia 
Tabla 1. Calificación derechos por zonas y departamentos

NIVELES DE RECONOCIMIENTO Y EXIGIBILIDAD DE DERECHOS RELACIONADOS CON LA ATENCIÓN DEL CONSUMO DE SPA DEPARTAMENTOS DE LA ZONA NORTE, CAFETERA Y CENTRO

\begin{tabular}{|c|c|c|c|c|c|}
\hline & $\begin{array}{c}\text { Derecho al } \\
\text { conocimiento e } \\
\text { información sobre SPA }\end{array}$ & $\begin{array}{c}\text { Derecho a la } \\
\text { intimidad y } \\
\text { protección de datos }\end{array}$ & $\begin{array}{c}\text { Derecho a la } \\
\text { prestación de } \\
\text { servicios de salud }\end{array}$ & $\begin{array}{c}\text { Derecho a la } \\
\text { inclusión y } \\
\text { participación }\end{array}$ & $\begin{array}{l}\text { Mecanismos de } \\
\text { exigibilidad de } \\
\text { derechos }\end{array}$ \\
\hline CESAR & Medio & Medio & Alto & Bajo & Medio \\
\hline MAGDALENA & Bajo & Bajo & Bajo & Bajo & Medio \\
\hline ATLÁNTICO & Alto & Bajo & Bajo & Medio & Medio \\
\hline GUAJIRA & Medio & Bajo & Alto & Medio & Medio \\
\hline SUCRE & Bajo & Bajo & Bajo & Bajo & Bajo \\
\hline BOLÍVAR & Medio & Medio & Medio & Medio & Bajo \\
\hline DISTRITO CARTAGENA & Medio & Alto & Medio & Bajo & Medio \\
\hline QUINDÍO & Alto & Bajo & Bajo & Bajo & Medio \\
\hline RISARALDA & Medio & Alto & Medio & Bajo & Bajo \\
\hline CALDAS & Bajo & Bajo & Bajo & Bajo & Bajo \\
\hline TOLIMA & Bajo & Medio & Bajo & Bajo & Alto \\
\hline META & Bajo & Bajo & Bajo & Bajo & Bajo \\
\hline SANTANDER & Medio & Medio & Medio & Bajo & Medio \\
\hline NORTE DE SANTANDER & Bajo & Bajo & Bajo & Bajo & Bajo \\
\hline CUNDINAMARCA & Medio & Medio & Bajo & Bajo & Bajo \\
\hline
\end{tabular}

Fuente: Elaboración propia

Inadecuada contratación de la red

Una de las barreras identificada, con mayor impacto en la gestión del riesgo, es la no contratación de los servicios incluidos a cargo de la UPC, con la red de prestadores del territorio; lo anterior conlleva a una subutilización de los servicios habilitados, un desequilibrio entre la demanda atendida y la oferta de servicios y finalmente, la contratación parcial de servicios, teniendo como resultado fragmentación de los servicios, a pesar de las tecnologías incluidas en el Plan Obligatorio de Salud que favorecen al usuario y cuya utilización no cuenta con seguimiento efectivo, ni nacional ni territorial, lo que conduce a que el derecho a la atención en salud de las personas que viven con este evento y el derecho a la salud en su conjunto no se estén garantizando.

Entre las grandes barreras identificadas esta lo relacionado con la contratación de la red de prestadores, ya que algunas de estas Instituciones Prestadoras de 
Servicios - IPS - no tiene ningún tipo de contrato con las aseguradoras y estas no muestran interés de hacerlo, de igual manera no se identifica ningún Ente de Control que los obligue a contratar el POS completo a pesar que la norma lo exija. Se continúa encontrando agendas cerradas, no entrega de medicamentos, negación de servicios y por ende el aumento del riesgo y daño en esta población y en especial para los preadolescentes y adolescentes que son los usuarios identificados con mayor riesgo de consumo de SPA en los departamentos participantes.

\section{Servicios no habilitados}

Otras de las barreras de contratación es el no cumplimiento del proceso de habilitación dado por la Resolución 2003 de 2014, por parte de las IPS; el incremento progresivo de las exigencias del Sistema Obligatorio de Garantía de Calidad - SOGCAS y el no acompañamiento por parte de las entidades competentes para apoyar a los prestadores de servicios de los territorios en este proceso y el cumplimiento de los estándares, así como el desarrollo de incentivos negativos sobre las EPS, cuando no cumplen con la contratación de lo definido en entonces POS, con entidades habilitadas.

Desarticulación entre el SGSSS y el Sistema de Bienestar Familiar (SBF)

El ICBF, para la contratación, invita a instituciones que cumplen con lineamientos, requisitos $\mathrm{y} / \mathrm{o}$ condiciones diferentes a las de habilitación en salud, lo que evidencia desarticulación entre el SGSSS y el SBF.

\section{Ausencia de sistema de información nacional}

Otras de las barreras identificadas es la falta de un sistema de información nacional, unificado con indicadores de seguimiento a los registros de las actividades, atenciones, remisiones, autorizaciones y negaciones, en las cuales se describa el tipo de usuarios y/ o poblacionales.

\section{Asimetría en derechos de población de regímenes especiales}

De otra parte, se pudo identificar que algunos regímenes especiales de salud, como docentes o militares, con alta incidencia en consumo de SPA, no cuentan con herramientas y capacidad técnica para la prestación de estos servicios especializados.

\section{DISCUSIÓN}

Como lo propone Nicolescu (1996), existen tres pilares que determinan la metodología de la investigación transdisciplinaria a saber: "Ios niveles de Realidad, la lógica del tercero incluido y la complejidad". Este proceso investigativo contempló diferentes niveles de realidad desde las ciencias sociales, económicas y de la salud para la comprensión del problema de acceso a los servicios de salud de las personas con consumo de SPA en el SGSSS colombiano; las instituciones prestadoras de servicios, los profesionales de la salud y los consumidores proponen diferentes niveles de percepción y enlazan entre sí los opuestos administrativos, de prestación de servicios, de aseguramiento, entre otros para proponer una aproximación a esta realidad en el país que permita desarrollar nuevas formas de superar las barreras de acceso identificadas.

Es importante recalcar que la metodología transdisciplinaria no es excluyente de las formas de estudio disciplinarias, sino que "fecunda estas disciplinas, proveyéndoles esclarecimientos nuevos e indispensables que no pueden ser producidos por la metodología disciplinaria" (Nicolescu, 1996), por ello la construcción conceptual de las barreras de acceso, así como las propuestas derivadas de ella se construyen con el aporte disciplinar de las ciencias sociales, de la salud, económicas y la bioética entre otras, y complementan los modelos biomédico, biopsicosocial y sociocultural del estudio de las adicciones, en tanto que se presente desde el enfoque de la prestación de servicios de atención integrales.

De los resultados encontrados en la investigación, descritos a continuación, se puede señalar que la ausencia desde lo práctico, de un enfoque de derechos humanos, incide tanto en la garantía efectiva de acceso a los servicios desde la perspectiva del derecho a la salud, como en los diferentes procesos administrativos que se desarrollan para la prestación adecuada de los mismos.

Las barreras administrativas, por impedir el acceso a servicios de salud, impiden en consecuencia, el ejercicio efectivo de este derecho, como bien lo han señalado algunos estudios en otros países, en relación con barreras desde la perspectiva de los consumidores, en cuanto a la posibilidad de iniciar tratamiento, acceder a este, u otras relacionadas con aspectos económicos, 
logísticos, administrativos, legal o cultural (Dols et. al., 2007)

Un enfoque basado en derechos, más allá desde lo teórico, que considera al usador como un sujeto en situación de vulneración, debe incidir en que el sistema lo vea como tal y no un paciente desde la perspectiva de sujeto pasivo frente al sistema, de forma que se entienda este grupo no como personas con necesidades que deben ser asistidas, sino sujetos con derecho a demandar determinadas prestaciones y conductas. En este sentido, tal como lo porpone Abramovich (2006: 2), el reconocimiento de tales derechos conlleva a la implementación de acciones efectivas derivadas de compromisos y obligaciones legales nacionales e internacionales en el marco de los tratados de derechos humanos, por lo que resulta mandatorio este enfoque en el desarrollo de políticas públicas.

En este mismo sentido, de los grupos focales, al evidenciarse el cumplimiento de derechos específicos en relación con los usuarios consumidores de SPA, se pudo evidenciar que nominalmente tenían referencia de los mismos, pero en relación con el contenido y la garantía efectiva, se encontraban serias disparidades.

Así, cuando se preguntaba por el consentimiento informado, se señala que se tenían los formatos respectivos, pero no se entendía, que este más allá de un procedimiento, es un derecho fundamental de los usuarios que desarrolla el principio por el respeto a la garantía por las normas y estándares éticos en los servicios de tratamiento, que incluye claramente una referencia a estándares éticos en la atención a los usuarios y sus familias afectadas por el consumo de SPA. De forma que, aún debe fortalecerse su comprensión de una simple autorización a un derecho fundamental, que a su vez garantiza el ejercicio del derecho a libertad personal, esto es, desde un enfoque bioético, como la garantía fundamental para el ejercicio pleno de la autonomía, que se fundamenta en la información necesaria para la toma de decisiones libres, comprensibles y fundamentas.

Es decir, que este proceso debe entenderse como un "proceso continuo, dialogístico (hablado), comunicativo, deliberativo y prudencial", voluntario, con información suficiente y comprensible, donde se involucra y determina la capacidad del sujeto para otorgarlo, o sus familiares o representantes, de forma que la toma de decisiones se hace en efecto tras un proceso completo de deliberación (Lorda \& Júdez, 2001). En este sentido el proceso de consentimiento informado, que responde a estándares éticos y jurídicos internacionales, se caracteriza por tomar en cuenta todos y cada uno de estos elementos.

Igualmente, en relación con el derecho a la protección de datos personales, como derecho que tiene origen en los principios éticos de privacidad $y$ confidencialidad, así entendidos como en el secreto profesional en el marco de las relaciones dentro del sistema de salud. Sin embargo, la mayoría de las legislaciones, con mayores o menores garantías, lo conciben como un verdadero derecho fundamental, así en el caso colombiano, la Ley 1581 de 2012, los datos en salud, o datos sensibles, son objeto de una protección especial, y en consecuencia los usuarios de los servicios del sistema de salud deben tener garantía de esta protección. Pero de los grupos focales y la revisión en algunas de los portales electrónicos de las IPS que prestan estos servicios, no se hacía referencia, por ejemplo, a la política específica de protección de datos personales.

Estos postulados se refieren de manera especial a la realización de principios en perspectiva bioética, especialmente: autonomía y responsabilidad, beneficencia, consentimiento, vulnerabilidad e integridad personal, privacidad y confidencialidad, no discriminación y no estigmatización, y responsabilidad social y salud.

Conforme a lo anterior, es determinante la necesidad de formular, validar y ejecutar un proceso de formación, apropiación y legitimación de un enfoque de derechos y de exigibilidad en el marco del sistema de salud que aborde las diferentes dimensiones del consumidor de sustancias psicoactivas como sujeto de derechos.

El no reconocimiento al consumidor como sujeto de derechos fomenta y agrava las situaciones de estigma, multidiscriminación, en tanto que "marca muy negativamente la vida tanto social laboral del individuo" (Arribas, 2001). Uno de los aportes en este trabajo es la caracterización territorial presentada en el capítulo anterior, la cual refleja una aproximación a la realidad de poblaciones invisibilizadas que presentan situaciones de consumo de SPA en contextos y vulnerabilidades 
diferenciados, lo cual convoca al llamado a considerar a los consumidores de SPA como un sujeto propiamente diferenciado para una intervención efectiva y aterrizada en todos los niveles y sectores gubernamentales y locales.

Si bien este es un fenómeno descrito desde las ciencias sociales, se refleja en un impacto directo en la atención en salud, siendo uno de los muchos vasos comunicantes identificados entre estos saberes. Ahora bien, la Corte Constitucional en diferentes pronunciamientos jurisprudenciales ha reconocido como sujeto de derechos a los usadores de sustancias psicoactivas, lo cual no se ve reflejado en la atención en salud de ellos.

Asimismo, conforme a los hallazgos de la caracterización territorial se observaron diferentes poblaciones como desplazados, afrodescendientes, desempleados, estudiantes, entre otros, que presentan diferentes procesos de consumo de SPA, lo cual convoca a que exista un reconocimiento de sujeto colectivo para avanzar en el país en la configuración de colectivos de consumidores, asociaciones de pacientes, de familias para la generación de procesos de gestión, análisis de los diferentes riesgos, vulnerabilidades, así como una incidencia en el logro de mejores condiciones de salud y de protección social en las políticas públicas.

El nuevo modelo de atención integral permite la posibilidad de procesos de autogestión, de interacción territorial, intersectorial conforme a los niveles de intervención individual y colectivo, para lo cual se necesita promover la consolidación de colectivos serios y espacios de participación efectivos.

Finalmente, dentro de limitaciones de esta investigación se tiene que el contacto con los participantes de las asistencias técnicas, en las que se aplicaron los instrumentos de recolección de información, se hizo vía institucional y sin recursos específicos para su desplazamiento, lo que dificultó la presencia de un amplio grupo de agentes, lo anterior pudo generar la subestimación de otro tipo de barreras

\section{Conclusiones}

Abordar, desde la transdisciplinariedad el conocimiento de las barreras de acceso a los servicios de salud a las personas con consumo de SPA en Colombia, ha permitido reconocer, dentro de los resultados las interacciones que estas barreras tienen entre fenómenos administrativos como el aseguramiento, derivados de las ciencias económicas; con la garantía del derecho a la atención de las personas con consumo, derivada de las ciencias sociales y la prestación de servicios de salud propiamente dichos relacionado con las ciencias de la salud, permitiendo la descripción compleja de este fenómeno, allí ciencias complejas como la bioética tienen una especial participación en el abordaje de estos fenómenos.

Los resultados en esta investigación permiten señalar que la ausencia del enfoque de derechos humanos es una barrera de gran trascendencia para la prestación efectiva de los servicios en relación con el consumo de SPA. Conforme a ello, es importante que se tomen dentro del sistema de salud, acciones reales para garantizar el cumplimiento y la exigibilidad de los derechos de los usuarios.

Acorde a lo anterior, las acciones deben ir encaminadas a la determinación de riesgo poblacional con enfoque diferencial, tendientes a mitigar la discriminación, multidiscriminación y estigma, y a garantizar la participación, la información completa y adecuada el cumplimiento de los derechos a la salud mental, a la prestación de los servicios de salud, consentimiento informado, a la libre elección de las Entidades Prestadoras de Salud y de las Instituciones Prestadoras de Salud para la promoción, prevención y tratamiento del consumo de SPA, derecho a la intimidad en materia médica, a la protección de la reserva del dato médico, y por supuesto, al reconocimiento a los consumidores como sujetos de derechos, que son aportados y complementados desde la transdisciplinariedad, esto es desde la mirada de la bioética y el derecho, especialmente.

El nuevo modelo de atención es una respuesta a las barreras de atención de los servicios de salud, los agentes de sistema lo reciben con gran expectativas y al mismo tiempo les genera desconfianza, dado al alto grado de vulnerabilidad de los usuarios y la vulneración de derechos que se vive actualmente en el SGSSS, derivada del no cumplimiento de la normatividad vigente. De esta forma, para dar cumplimiento al MIAS se requiere de un sistema de Inspección, Vigilancia y Control que permita la toma de decisiones informadas por parte de las 
entidades competentes, así como el seguimiento territorial de estas acciones.

Se considera que los procesos de asistencia técnica, formación y capacitación en los cambios de la normatividad vigente deberían ser de obligatoriedad para los agentes directos del Sistema de Salud y gestionados desde las entidades departamentales, Alcaldías y entidades competentes, lo cual ha de incidir en la articulación total entre las instituciones que trabajan la problemática de las SPA y así incidir en la capacidad de construir un trabajo en red efectivo para la implementación de las RISS.

Por lo otro lado, acorde con la revisión de programas, literatura académica, planes, políticas y los resultados de las asistencias técnicas, es importante repensar el sistema de incentivos en la prestación de servicios relacionados con el consumo de SPA, para que contribuyan al fortalecimiento de la calidad en la prestación de servicios. Desde una revisión ética de su determinación, los mismos deben responder a una serie de criterios que se han de tomar en consideración al contexto del sistema de salud en particular. Las bondades de los mismos, dependen del compromiso, las capacidades institucionales y la voluntad política de los agentes implicados en la medida que se genere un consenso en la necesidad pertinencia de establecer y aplicar una política de incentivos en este servicio de manera particular.

\section{Agradecimientos}

Este artículo se enmarca y presenta resultados del proyecto de investigación "Estudio de los servicios de tratamiento a las adicciones fundamentadas en el sistema general de seguridad social en salud y normatividad adicional aplicable en Colombia", financiado por la oficina de Colombia de UNODC, llevado a cabo entre junio y diciembre de 2016. Agradecemos de manera especial el apoyo técnico del comunicador social Diego Alexander Rojas Pulido, a la enfermera Teresa Rodríguez y a la abogada Carolina Duque Núñez, consultores dentro del proyecto.

\section{REFERENCIAS}

Abramovich, V. (2006). Una aproximación al enfoque de derechos. Revista de la CEPAL, 88, 35.
Arribas, J. R. (2001). Proceso de construcción de un estigma: la exclusión social de la drogadicción. Nómadas. Revista Crítica de Ciencias Sociales y Jurídicas, 4(2), 233-243.

Camarotti, A. C. \& A. L. Kornblit (2015) Abordaje integral comunitario de los consumos problemáticos de drogas: construyendo un modelo / A comprehensive community approach to problematic drug use: building a model. Salud Colectiva, 211.

Congreso de la República. (31 de julio de 2012). Por la cual se dictan normas para garantizar la atención integral a personas que consumen sustancias psicoactivas y se crea el premio nacional "Entidad comprometida con la prevención del consumo, abuso y adicción a sustancias psicoactivas". [Ley 1566 de 2012]. DO 48508. Recuperado http://wsp.presidencia.gov.co/Normativa/Leyes/Documents/ley15 6631072012.pdf.

Congreso de la República. (17 de octubre de 2012). Por la cual se dictan disposiciones generales para la protección de datos personales. [Ley Estatutaria 1581 de 2012] DO 48587. Recuperado de: http://www.secretariasenado.gov.co/senado/basedoc/ley 158120 12.html

Corte Constitucional. (11 de octubre de 2012). Sentencia, T. 796. [MP María Victoria Calle Correa].

Corte Constitucional. (13 de marzo de 2014). Sentencia, T. 153. [MP Mauricio González Cuervo].

Dols, S. T., Gras, T. S., Rodrigo, M. T., Infer, A. V., Moya, R. A., \& Zurián, J. V. (2007). Barreras de accesibilidad al tratamiento: diferencias entre adictos a la heroína y adictos a la cocaína de la Comunidad Valenciana. Trastornos adictivos, 9(3), 206-214.

Ministerio de Salud y Protección Social. (14 de mayo de 2013). Plan Decenal de Salud Pública 2012-2021: La salud en Colombia la construyes tú. [Resolución 1481 de 2013]. Recuperado de: https://www.minsalud.gov.co/sites/rid/Lists/BibliotecaDigital/RIDE/ DE/DIJ/resolucion-1841-de-2013.pdf

Ministerio de Salud y Protección Social. (28 de mayo de 2014). Por la cual se definen los procedimientos y condiciones de inscripción de los Prestadores de Servicios de Salud y de habilitación de servicios de salud. [Resolución 2003 de 2014]. Recuperado de: https://www.minsalud.gov.co/Normatividad_Nuevo/Resoluci\%C3\% B3n\%202003\%20de\%202014.pdf

Ministerio de Salud y Protección Social. (24 de febrero de 2015). Por la cual se dictan disposiciones en relación con la Gestión de la Salud Pública y se establecen directrices para la ejecución, seguimiento y evaluación del Plan de Salud Pública de Intervenciones Colectivas PIC [Resolución 0518 de 2015]. Recuperado de: https://www.minsalud.gov.co/Normatividad_Nuevo/Resoluci\%C3\% B3n\%200518\%20de\%202015.pdf

Musto David F., La enfermedad americana. Orígenes del control antinarcóticos en EU, CEl, Ediciones Uniandes, Tercer Mundo Editores, Bogotá, 1993, pp. 248-254

Naomar, A.-F. (2006) Complejidad y Transdisciplinariedad en el Campo de la Salud Colectiva: Evaluación de Conceptos y Aplicaciones / 
Complexity and Transdisciplinarity in the Collective Health Field: Concepts' Evaluation and Applications. Salud colectiva, pp. 123.

Nicolescu, N. (1996). La Transdisciplinariedad. Manifiesto. Francia: Ediciones du Rocher, Versión en español Norma Núñez y Dentón.

Organización de las Naciones Unidas (2016). Sesión Especial de la Asamblea General de las Naciones Unidas UNGASS. A/RES/S-30/1, Nuestro compromiso conjunto de abordar y contrarrestar eficazmente el problema mundial de las drogas, Resolución aprobada por la Asamblea General el 19 de abril de 2016

Peláez, I. A., \& Romaní, O. (2016). La encrucijada de la adicción. Distintos modelos en el estudio de la drogodependencia. Health and Addictions/Salud y Drogas, 16(2), 115-126.

Ramis Andalia, R. M. (2007) Complejidad y salud en el siglo XXI. (Spanish). Complexity and healthcare in the 21st century. (English), 33, 1-11.

Sotolongo y Delgado. La epistemología hermenéutica de segundo orden En Publicación La revolución contemporánea del saber y la complejidad social. Hacia unas ciencias sociales de nuevo tipo. Buenos Aires : Consejo Latinoamericano de Ciencias Sociales CLACSO, 2006.

Simón Lorda, P., \& Júdez Gutiérrez, J. (2001). Consentimiento informado. Medicina clínica, 117(3), 99-106.

\section{GLOSARIO}

EPS Entidades Promotoras de Salud

ICBF Instituto Colombiano de Bienestar Familiar

IPS Instituciones prestadoras de Servicios de Salud

MIAS Modelo Integral de la Atención en Salud

MSPS Ministerio de Salud Y Protección Social de Colombia

POS Plan Obligatorio de Salud, también denominado en ocasiones plan de beneficios a cargo de la UPC

SBF Sistema de Bienestar Familiar

SGSSS Sistema General de Seguridad Social en Salud

SOGCAS Sistema Obligatorio de garantía de la Calidad de la Atención en Salud

SPA Sustancias psicoactivas

UNODC Oficina de las Naciones Unidas Contra las Drogas y el Delito 